\title{
CARIOSTATIC EFFICACY AND CHILDREN ACCEPTANCE OF NANO-SILVER FLUORIDE VERSUS SILVER DIAMINE FLUORIDE: A RANDOMIZED CONTROLLED CLINICAL TRIAL
}

\author{
Zuhair Al-Nerabieah', Ettihad Abo Arrag ${ }^{1}$, Anas Rajab² \\ 'Department of Pediatric Dentistry, Damascus University, Syria \\ ${ }^{2}$ Department of Organic Chemistry, Syrian Private University, Syria
}

\begin{abstract}
INTRODUCTION: Untreated caries in primary teeth is considered one of the most common epidemic problems around the world.

ОвJECTIVEs: The aim of this study was to evaluate the cariostatic efficacy and patient acceptance of nano-silver fluoride (NSF) compared to silver diamine fluoride (SDF) 38\% in primary teeth in preschool children.

MATERIAL AND METHODS: 119 children, with a total of 244 active dentin lesions were randomly assigned into two groups: NSF group included 60 children with 122 lesions, and SDF group consisted of 59 children with 122 lesions. Outcome assessments were performed at 3 weeks and 6 months after treatment using Nyvad criteria to assess the activity of carious lesions. Wong-Baker faces pain rating scale was used to assess children acceptance for the treatment. RESULTs: After 3 weeks, 94 (77\%) lesions in NSF group were arrested, while 110 (90\%) lesion were arrested in SDF group $(p>0.05)$. After six months, the arrest rate in NSF and SDF groups was $67.2 \%$ and $79.5 \%$, respectively $(p>0.05)$. However, the arrest rate of single surface lesions on anterior teeth was higher after six months in both groups $(p<0.05)$. Furthermore, patient acceptance scores were not statistically different in both groups $(p>0.05)$. ConcLusions: Both SDF and NSF were effective in arresting carious dentine lesions and were accepted by preschool children.
\end{abstract}

KEY WORDS: nano-silver fluoride, silver diamine fluoride, cariostatic agent, dental caries, children.

J Stoma 2020; 73, 3: 100-106

DOI: https://doi.org/10.5114/jos.2020.96939

\section{INTRODUCTION}

Untreated caries in both primary and permanent teeth are considered one of the most common epidemic problems around the world. Whether the reason was, the higher expenses or the lower cooperation level, young children living in low income countries suffer a higher incidence of having untreated carious lesions $[1,2]$.
Atraumatic restoration technique was intended to resolve caries issues in a deprived population. However, this technique requires instruments to excavate the carious lesion and restorative materials and cooperation [3].

Lately, arresting caries using silver diamine fluoride (SDF) has been proved to be an effective way to treat cavitated lesion $[4,5]$. Nevertheless, silver forms a black discoloration of carious lesion, which can be deliberated as a main downside of its use, specifically on anterior teeth $[6,7]$.

JOURNAL OF STOMATOLOGY CZASOPISMO STOMATOLOGICZNE

AdDRESS FOR CORRESPONDENCE: Dr. Zuhair Al-Nerabieah, Department of Pediatric Dentistry, Damascus University, Syria, e-mail: Zuhairmajid@gmail.com 
A researcher team from Brazil have chemically synthesized a formulation of nano-silver, chitosan, and fluoride. This NSF product was effective in arresting caries without blacking the dentin tissue [8]. Targino et al. evaluated the cytotoxicity of different concentrations of nano-silver solutions that ranged from (1\% to 5\%), and they found no toxic effect for erythrocytes [9].

To the best of our knowledge, there was only one in vivo study that have evaluated the efficacy of nano-silver fluoride (NSF) versus SDF in arresting caries in primary molars [10].

Therefore, this study aim was to investigate the cariostatic efficacy and patient acceptance of NSF compared to SDF $38 \%$ in both anterior and posterior primary teeth in preschool children.

\section{MATERIAL AND METHODS}

\section{PREPARATION OF NANO-SILVER FLUORIDE}

To prepare nano-silver particles, Dos Santos et al. chemical experiment was followed [8], and the formation of nano-silver particles (AgNPs) was noted immediately by the displayed brown color of the solution, which was in correspondence with other studies [11-13].

At the end of the chemical synthesis of AgNPs, sodium fluoride $(\mathrm{NaF})$ was added to improve the stability and the cariostatic efficacy of the solution (10,000 ppm). NSF was kept in a dark black PET bottle at $4^{\circ} \mathrm{C}$, until further use.

The shape and size of the synthesized AgNPs were analyzed using scanning electron microscopy (SEM) (VEGA second generation, TESCAN, Czech Republic) and revealed that AgNPs were spherical with an average particle size of $4 \mathrm{~nm}$ (Figure 1).

Furthermore, SDF used in this study was Advantage Arrest $^{\oplus}$ silver diamine fluoride 38\% (Elevate Oral Care, West Palm Beach, USA).

\section{CLINICAL TRIAL}

This study design was a randomized, double blind, clinical trial with an active control group. Ethical approval was obtained from the University Research Ethics Committee, and the trial was registered in the ANZCTR, with following ID: ACTRN12618001865202. The CONSORT statement for reporting randomized controlled trials was applied in this research. The study took a place in a single day care center between the period of August 2018 and May 2019. Parents of enrolled children have signed an informed consent before the initiation of the study.

\section{SAMPLE SIZE CALCULATION}

The sample size was determined with ClinicCal Website (University of Indianapolis, USA). The $\alpha$ and $\beta$ level were set at 0.05 and $80 \%$, respectively. Based on previous studies [10], it was estimated that 80 carious lesions in each group were sufficient to show an effect size (0.4) in the average proportion of arrested caries.

\section{PARTICIPANTS ENROLLMENT}

Clinical examination to enroll eligible children was performed by an experienced investigator (AZ) using a disposable blunt probe and a headlight. Inclusion criteria were as following: co-operative healthy children aged 3 to 5 years with at least one active dentin lesion based on Nyvad criteria, with an absence of spontaneous pain and sensitivity to percussion [14]. The tooth was excluded if it had pulp exposure, abscess/fistula, or discoloration related to tooth non-vitality.

\section{RANDOMIZATION}

119 children, with a total of 244 active caries lesions met the inclusion criteria and were enrolled into this study. Each child was appointed to one intervention arm to prevent the viable cross-over effect of the NSF and SDF (38\% Advantage Arrest, Elevate Oral Care, USA). Therefore, to balance the baseline numbers of active caries between the two groups, participants were first classified into two strata according to their active caries lesions (1-2 lesions or $\geq 3$ lesions). Then, children were randomly allocated to one of the groups, with $1: 1$ ratio as shown in the CONSORT flowchart in Figure 2.

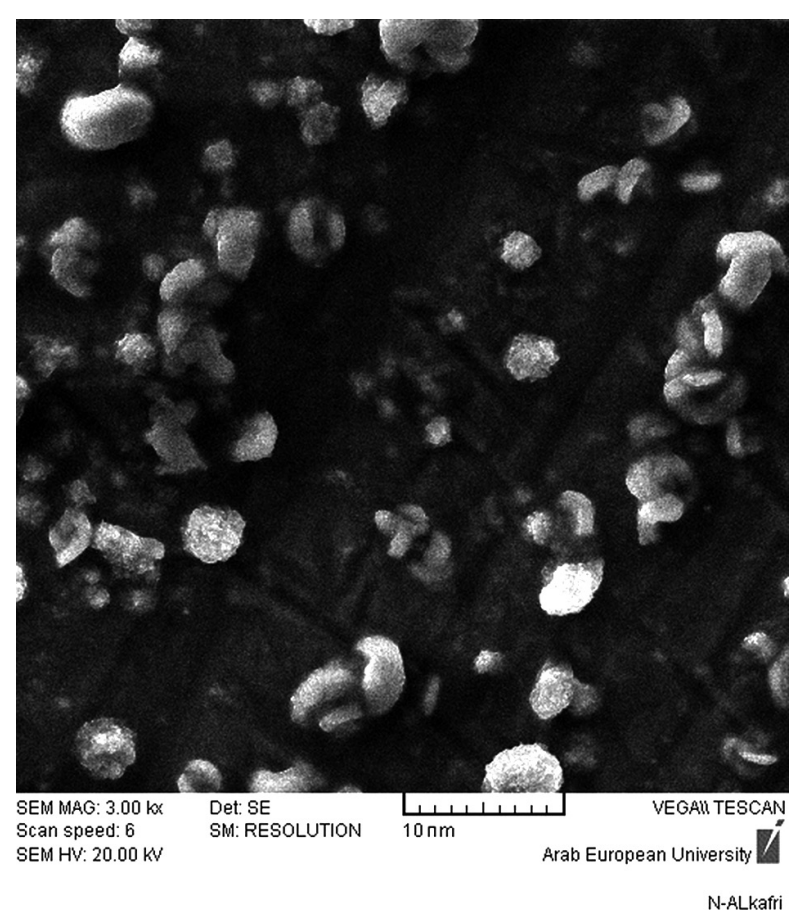

FIGURE 1. Scanning electron microscopy (SEM) of nanosilver particles 


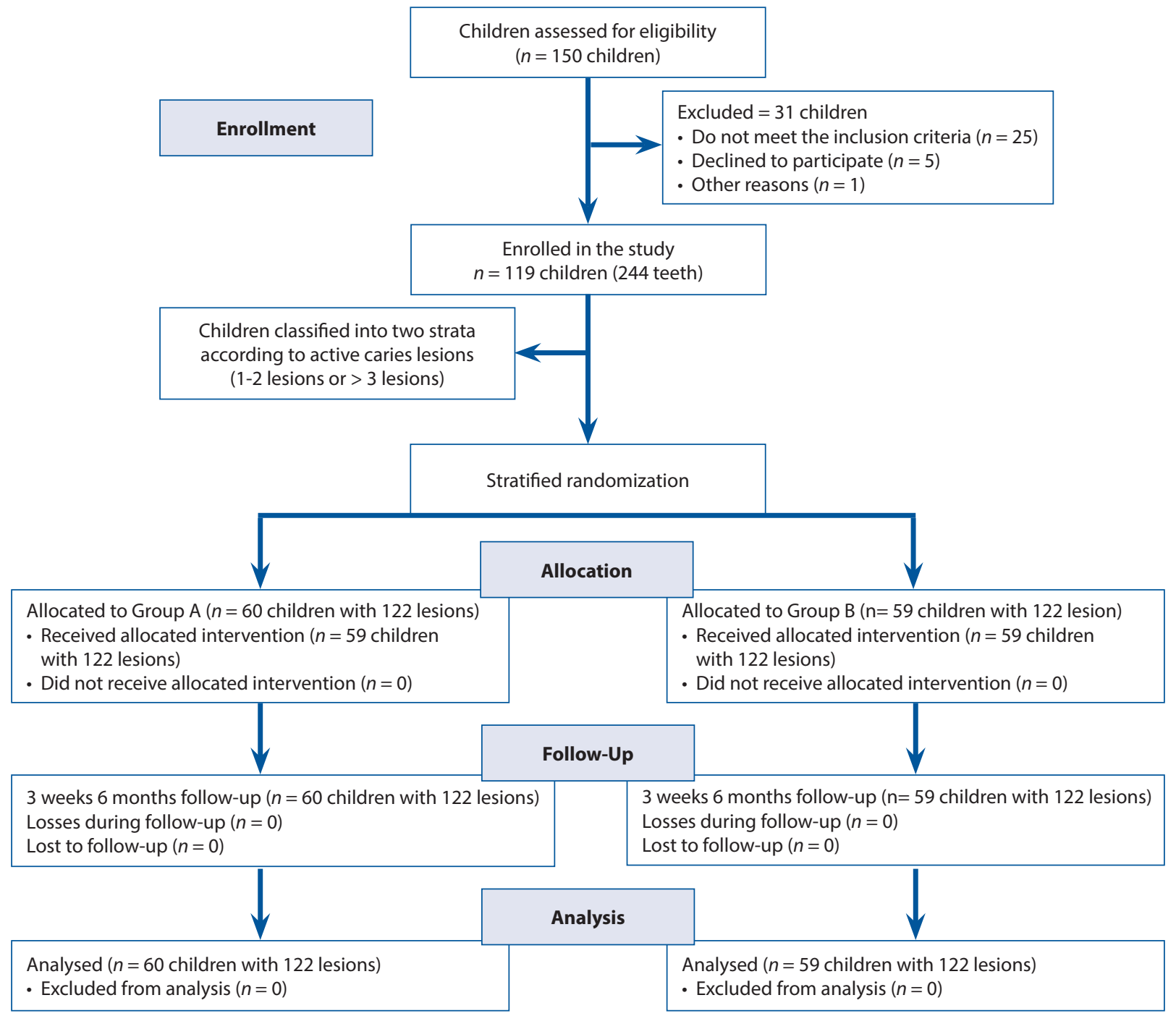

FIGURE 2. The CONSORT flowchart

\section{CLINICAL INTERVENTION}

During the first visit, decay, extracted due to caries, filled tooth (deft) index was scored for all children to assess oral hygiene status. Then, caries activity assessment was carried out using visual and tactile inspection, according to Nyvad criteria [15]. Teeth with cavitated lesions, where the surface felt soft on gentle probing, were considered active lesion, and enrolled in the study. At the end of the first visit, toothbrushes and written oral hygiene instruction were distributed among parents and guardians of participants.

In the next visit, both intervention agents (NSF/ SDF) were applied in the same manner in accordance to the University of California San Francisco (UCSF) protocol [16]. Firstly, cleaning of the affected tooth surfaces was done by a disposable micro-brush (Microbrush International, Grafton, USA) for at least 30 seconds and then dried with cotton gauze sponges. No effort was made to excavate the infected dentin tissue before the application of the agent. Gum was protected with petroleum jelly, and isolation was carried out using cotton rolls.

After isolation, a single drop of one of the agents (NSF/SDF) was applied into the lesion surface with a disposable micro-brush for at least two minutes. After that, site of application was covered with petroleum jelly. After finalizing the application process, children satisfaction and acceptance was scored using Wong-Baker ${ }^{\circledR}$ faces pain rating scale (WBFPRS). The scale shows a series of faces, ranging from a happy face at 0 (no hurt) to a crying face at 10 (Figure 3 ).

After that, instructions were given to the teachers that children participating in the trial were not allowed to eat or drink for an hour after the application of agents [17].

Outcome assessment of the lesions was carried out using a visual and tactile assessment, which was performed at 3 weeks and 6 months after treatment, using Nyvad criteria to assess carious lesions activity. The assessment was achieved by two blinded calibrated investigators us- 
ing a blunt probe and artificial light. Shiny lesions that felt hard on gentle probing were considered arrested cavities. In the same session, after assessing caries activity for all children, $10 \%$ of the total sample was randomly re-evaluated to monitor intra-examiner reproducibility.

\section{BLINDING}

Participants were blinded towards agents used in the study by using two identical squeezable plastic droppers with tag codes A and B. Also, investigators and biostatistician were blinded from the meaning of codes.

\section{STATISTICAL ANALYSIS}

Normal distribution of quantitative data was tested by Kolmogorov-Smirnov test. For data analysis, two sample $t$-test was used for means of comparisons. Intragroup comparisons were made using chi-square test with 95\% confidence interval. Fisher's exact test was used for intergroup comparison. Statistical tests were performed with a margin of error of $5.0 \%$. Statistics were calculated using Statistical Package for Social Sciences (SPSS) version 25.0 (IBM, Chicago, IL, USA). Intra-rater reliability and inter-examiner agreement for caries assessment was calculated by Cohen's $\kappa$ test. The $\kappa$ for intra-rater reliability were 0.85 and 0.90 . The $\kappa$ for inter-examiner agreement was 0.87 .

\section{RESULTS}

119 children (50 males and 69 females) were enrolled into the study, with a mean age of 3.6 and 3.9 years for NSF and SDF groups, respectively. Table 1 shows descriptive analysis regarding deft index, number of teeth, frequency of type, and site of the lesion for each group.

The frequency of type and site of lesions in each group were not identical, since some children had more than one lesion and each child were assigned to one intervention only. However, as shown in Table 1, there were no statistically significant differences between the two groups $(p>0.05)$. After six months, all participants were presented, and no loss of teeth was recorded.

In the first follow-up period, after 3 weeks, 94 (77\%) lesions in NSF group were arrested, while 110 (90\%) lesion were arrested in SDF group $(p>0.05)$. After six months, the arrest rate in NSF and SDF groups was $67.2 \%$ and $79.5 \%$, respectively $(p>0.05)$ (Table 2 ).

Furthermore, the arrest rate of single surface lesions and anterior teeth was statistically significant in NSF and SDF group after six months $(p<0.05)$ (Table 3 ).

Regarding children acceptance, $85 \%$ of the patients in NSF group have reported grade 0 (no hurt) in the WBFPRS, and $91.5 \%$ of the patients in SDF group have reported the same grade. Only two children (3\%)

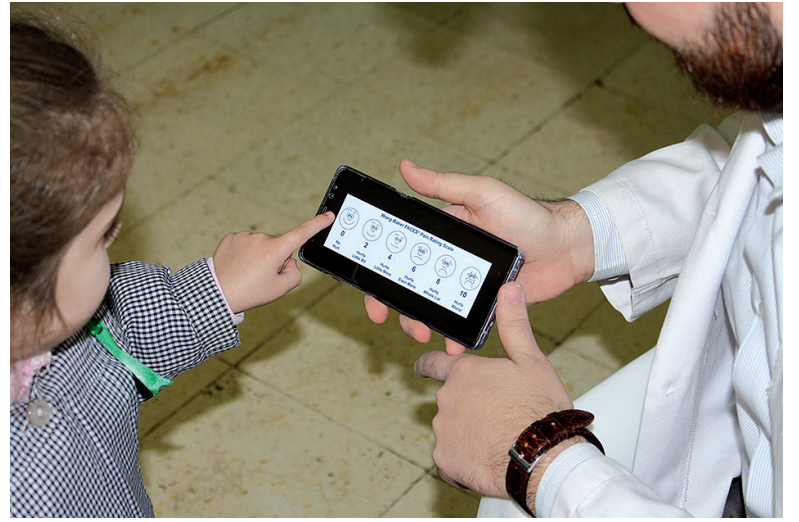

FIGURE 3. Evaluation of patients' acceptance after the treatment using Wong-Baker ${ }^{\circledR}$ faces pain rating scale

TABLE 1. Analysis of demographic variables and frequency of type and site of active lesions

\begin{tabular}{|c|c|c|c|}
\hline Variables & NSF group & SDF group & p-value ${ }^{*}$ \\
\hline Children enrolled & 60 & 59 & $0.97^{* *}$ \\
\hline Male & 22 & 28 & $0.83^{* *}$ \\
\hline Female & 38 & 31 & $0.90^{* *}$ \\
\hline Age (mean $\pm S D)$ & $3.6 \pm 0.5$ & $3.9 \pm 0.4$ & $0.68^{* *}$ \\
\hline Deft (mean $\pm S D)$ & $4.9 \pm 0.7$ & $5.1 \pm 0.5$ & $0.126^{* *}$ \\
\hline Active lesion & 122 & 122 & \\
\hline \multicolumn{4}{|l|}{ Type of lesion } \\
\hline Single surface (\%) & $40(33 \%)$ & $36(30 \%)$ & \multirow{2}{*}{$0.471^{* *}$} \\
\hline Multiple surface (\%) & $82(67 \%)$ & $86(70 \%)$ & \\
\hline \multicolumn{4}{|l|}{ Site of lesion } \\
\hline Anterior & $49(40 \%)$ & $51(42 \%)$ & \multirow{2}{*}{$0.866^{* *}$} \\
\hline Posterior & $73(60 \%)$ & $71(58 \%)$ & \\
\hline
\end{tabular}

TABLE 2. Evaluation of caries arrest rate at 21 days and 6 months

\begin{tabular}{|c|c|c|c|c|}
\hline Variables & NSF group & SDF group & $\chi^{2}$ & $p$-value* \\
\hline \multicolumn{5}{|l|}{21 days } \\
\hline Arrested & 94 (77\%) & $110(90 \%)$ & \multirow{3}{*}{1.254} & \multirow{3}{*}{$0.263^{* *}$} \\
\hline Active & $28(23 \%)$ & $12(10 \%)$ & & \\
\hline $95 \% \mathrm{Cl}^{\mathrm{a}}$ & $0.622-0.942$ & $0.741-1.086$ & & \\
\hline \multicolumn{5}{|l|}{6 months } \\
\hline Arrested & $82(67.2 \%)$ & 97 (79.5\%) & \multirow{3}{*}{1.257} & \multirow{3}{*}{$0.262^{* * *}$} \\
\hline Active & $40(32.8 \%)$ & $25(20.5 \%)$ & & \\
\hline $95 \% \mathrm{Cl}^{\mathrm{a}}$ & $0.534-0.834$ & $0.644-0.969$ & & \\
\hline
\end{tabular}

a95\% confidence interval for average proportion of arrested caries and difference in average proportion of arrested caries, ${ }^{*}$ chi-square, ** ${ }^{*}$ statistically not significant

in NSF group have reported grade 2 (hurts a little more). However, this difference between both groups was not statistically significant $(p>0.05)$ (Table 4$)$. 
TABLE 3. Intra-group evaluation of caries arrest rate according to type and site of the lesion after 6 months

\begin{tabular}{|c|c|c|c|}
\hline \multirow{2}{*}{ Treatment/Variables } & \multicolumn{2}{|c|}{6 months } & \multirow{2}{*}{$p$-value* } \\
\hline & Arrested & Active & \\
\hline \multicolumn{4}{|l|}{ Nano-silver fluoride } \\
\hline \multicolumn{4}{|l|}{ Type of lesion } \\
\hline Single surface & $32(80 \%)$ & $8(20 \%)$ & \multirow{2}{*}{$0.041^{* *}$} \\
\hline Multiple surface & $50(61 \%)$ & $32(39 \%)$ & \\
\hline \multicolumn{4}{|l|}{ Site of lesion } \\
\hline Anterior & $41(83 \%)$ & $8(17 \%)$ & \multirow{2}{*}{$0.017^{* *}$} \\
\hline Posterior & $53(72 \%)$ & $30(28 \%)$ & \\
\hline \multicolumn{4}{|l|}{ Silver diamine fluoride } \\
\hline \multicolumn{4}{|l|}{ Type of lesion } \\
\hline Single surface & $33(91 \%)$ & $3(9 \%)$ & \multirow{2}{*}{$0.047^{* *}$} \\
\hline Multiple surface & $64(78 \%)$ & $22(12 \%)$ & \\
\hline \multicolumn{4}{|l|}{ Site of lesion } \\
\hline Anterior & $46(86 \%)$ & $6(14 \%)$ & \multirow{2}{*}{$0.040^{* *}$} \\
\hline Posterior & $51(63 \%)$ & $20(37 \%)$ & \\
\hline
\end{tabular}

*Fisher exact test, **statistically significant

TABLE 4. Comparison of Wong-Baker faces pain rating scale scores between NSF and SDF groups

\begin{tabular}{|c|c|c|c|}
\hline $\begin{array}{l}\text { Wong-Baker } \\
\text { faces scale }\end{array}$ & $\begin{array}{c}\text { NSF group, } \\
n(\%)\end{array}$ & $\begin{array}{c}\text { SDF group, } \\
n(\%)\end{array}$ & $p$-value ${ }^{*}$ \\
\hline Grade 0 & $51(85 \%)$ & $54(91.5 \%)$ & \multirow{7}{*}{$0.765^{* *}$} \\
\hline Grade 1 & $7(12 \%)$ & $5(8.5 \%)$ & \\
\hline Grade 2 & $2(3 \%)$ & 0 & \\
\hline Grade 3 & 0 & 0 & \\
\hline Grade 4 & 0 & 0 & \\
\hline Grade 5 & 0 & 0 & \\
\hline Mean rank & 11.75 & 10.98 & \\
\hline
\end{tabular}

\section{DISCUSSION}

This study was the first trial to assess the cariostatic efficacy and children acceptance of NSF compared to SDF in both anterior and posterior primary teeth in preschool children.

The age group in this trial was chosen to be between 3 and 5 years old, since preschoolers living in underprivileged communities with limited access to healthcare, suffer from a high incidence of untreated carious lesions $[1,18,19]$.

Recently, it was concluded that SDF was the material of choice (gold standard) for arresting dentin caries in deciduous teeth $[4,20]$. Thus, it was chosen in this trial as an active control group.
Nyvad criteria have been investigated thoroughly, and it was reported that these criteria have a good validity and reliability in evaluating caries activity in primary teeth $[15,21,22]$.

Results of this trial showed that both NSF and SDF were effective in arresting dentin carious lesion in primary teeth. The arrest rate for SDF group was higher than that in NSF group in both follow-up periods (3 weeks, 6 months). However, this difference was not statistically significant $(p>0.05)$. A higher arrest rate could be related to that fact that SDF $38 \%$ contains higher concentrations of active components ( 5 times $\mathrm{AgNO}_{3}$, 4 times $\mathrm{NaF})[23,24]$.

Dos Santos et al., the team who discovered this novel material (NSF), has reported similar arrest rate in their study after one-year of follow-up [8]. The mechanism of action of this product is related to the synergetic effect of NSF components (AgNPs, chitosan, and fluoride).

Nano-silver particles play a huge role in this process, since it was shown in literature that AgNPs possess a bactericidal and bacteriostatic effect against pathogens stains, which induce caries in the oral cavity, such as Streptococcus mutans and Lactobacillus acidophilus $[25,26]$.

Another unique feature of AgNPs show that those particles do not oxidize and as a result, NSF does not cause black discoloration in carious lesions [27]. As a matter of fact, this was observed in this trial, as all arrested lesion in SDF group have turned into black, while lesion arrested by NSF have not.

Furthermore, chitosan also owns an antimicrobial effect, and in this solution acts as a protective agent to stabilize and prevent AgNPs from aggregating [28].

However, after reviewing the literature, there was only one trial that compared the efficacy of NSF to SDF in arresting dentin carious lesions in primary molars only. Even though Tirupathi et al. reported that there was no significant difference between NSSF and SDF groups (which was similar to our results), but the arrest rate of nano-silver incorporated sodium fluoride (NSSF) group in Tirupathi's study was higher after one year of follow-up (77\%) [10]. This difference could be attributed to the children age in that trial being older and resided in an optimally fluoridated area. In contrary, children in this trial were 3-5 years old, living in a non-fluoridated community. Also, another possible reason is that silver nitrate concentration in the Tirupathi's study was higher (5\%) in the NSSF product, whereas in this trial was 3\%.

The present study showed that anterior teeth and single surface lesions showed higher arrest rates in comparison to posterior teeth and multiple surface lesions. This result was statistically significant and can be attributed to the fact that molars and multiple surface lesions are more prone to plaque accumulation and food impaction. Also, these lesions are harder to clean by the parents of preschool children [20,29].

Regarding children acceptance, the majority of participants have scored grade 0 in WBFPRS, which indi- 
cates a higher acceptance and a lower discomfort levels of the treatment. This high acceptance rate might be correlated with the fact that the application process of NSF/ SDF is simple, fast, and non-invasive. Others have reported similar results for children acceptance after SDF application [30].

Limitation of this study was that the process of blinding of the investigators might be compromised, since SDF arrested lesions has a black discoloration. Furthermore, the follow-up period could be considered relatively short.

Further studies with longer follow-up periods in multiple day care centers are required to assess the best protocol for NSF application.

\section{CONCLUSIONS}

Within the limitation of this study, we can confirm that NSF and SDF are both effective and accepted by preschool children, when used as a cariostatic agent for arresting cavitated active carious lesions in primary teeth in a non-fluoridated community. However, NSF might be more preferred on anterior teeth since this agent does not cause discoloration.

\section{ETHICAL STATEMENT}

All procedures performed in studies involving human participants were in accordance with the ethical standards of the institutional and/or national research committee, and with the 1964 Helsinki Declaration and its later amendments or comparable ethical standards. Informed consent was obtained from a parent or guardian of every participant included in the study.

\section{ACKNOWLEDGEMENT}

The authors are grateful to the faculty of pharmacy at the Syrian Private University for experimental support. Special thanks for Muaz AlKhouli and Salma AlNesser for serving as examiners in the trial. We thank Safaa Shehabi for her assistance with the SEM measurements.

\section{CONFLICT OF INTEREST}

The authors declare no potential conflicts of interest with respect to the research, authorship, and/or publication of this article.

\section{References}

1. Kassebaum NJ, Bernabé E, Dahiya M, Bhandari B, Murray CJL, Marcenes W. Global burden of untreated caries: a systematic review and metaregression. J Dent Res 2015; 94: 650-658.

2. Folayan MO, El Tantawi M, Aly NM, et al. Association between early childhood caries and poverty in low and middle income countries. BMC Oral Health 2020; 20: 8.
3. Frencken JE. Atraumatic restorative treatment and minimal intervention dentistry. Br Dent J 2017; 223: 183.

4. Crystal YO, Niederman R. Evidence-based dentistry update on silver diamine fluoride. Dent Clin 2019; 63: 45-68.

5. Gao SS, Zhao IS, Hiraishi N, et al. Clinical trials of silver diamine fluoride in arresting caries among children: a systematic review. JDR Clin Transl Res 2016; 1: 201-210.

6. Crystal YO, Niederman R. Silver diamine fluoride treatment considerations in children's caries management. Pediatr Dent 2016; 38: 466-471.

7. Patel J, Anthonappa RP, King NM. Evaluation of the staining potential of silver diamine fluoride: in vitro. Int J Paediatr Dent 2018; 28: 514-522.

8. Dos Santos Jr VE, Vasconcelos Filho A, Targino AGR, et al. A new "Silver-Bullet" to treat caries in children - Nano Silver Fluoride: a randomised clinical trial. J Dent 2014; 42: 945-951.

9. Targino AGR, Flores MAP, Dos Santos VE, et al. An innovative approach to treating dental decay in children. A new anti-caries agent. J Mater Sci Mater Med 2014; 25: 2041-2047.

10. Tirupathi S, Nirmala S, Rajasekhar S, Nuvvula S. Comparative cariostatic efficacy of a novel Nano-silver fluoride varnish with $38 \%$ silver diamine fluoride varnish a double-blind randomized clinical trial. J Clin Exp Dent 2019; 11: e105.

11. Modrzejewska Z, Zarzycki R, Sielski J. Synthesis of silver nanoparticles in a chitosan solution. Prog Chem Appl Chitin Deriv 2010; 15: 63-72.

12. Wei D, Sun W, Qian W, Ye Y, Ma X. The synthesis of chitosanbased silver nanoparticles and their antibacterial activity. Carbohydr Res [Internet] 2009; 344: 2375-2382. Available from: http:// dx.doi.org/10.1016/j.carres.2009.09.001

13. Jayakumar R, Menon D, Manzoor K, Nair SV, Tamura H. Biomedical applications of chitin and chitosan based nanomaterials - a short review. Carbohydr Polym 2010; 82: 227-232.

14. Nyvad B, Fejerskov O. Assessing the stage of caries lesion activity on the basis of clinical and microbiological examination. Community Dent Oral Epidemiol 1997; 25: 69-75.

15. Nyvad B, Baelum V. Nyvad criteria for caries lesion activity and severity assessment: a validated approach for clinical management and research. Caries Res 2018; 52: 397-405.

16. Horst JA, Ellenikiotis H, Milgrom PM, Committee USCA. UCSF protocol for caries arrest using silver diamine fluoride: rationale, indications, and consent. J Calif Dent Assoc 2016; 44: 16.

17. Milgrom P, Horst JA, Ludwig S, et al. Topical silver diamine fluoride for dental caries arrest in preschool children: a randomized controlled trial and microbiological analysis of caries associated microbes and resistance gene expression. J Dent 2018; 68: 72-78.

18. Psoter WJ, Pendrys DG, Morse DE, Zhang H, Mayne ST. Associations of ethnicity/race and socioeconomic status with early childhood caries patterns. J Public Health Dent 2006; 66: 23-29.

19. Brown LJ, Wall TP, Lazar V. Trends in untreated caries in primary teeth: of children 2 to 10 years old. J Am Dent Assoc 2000; 131: 93-100.

20. Trieu A, Mohamed A, Lynch E. Silver diamine fluoride versus sodium fluoride for arresting dentine caries in children: a systematic review and meta-analysis. Sci Rep 2019; 9: 1-9.

21. Séllos MC, Soviero VM. Reliability of the Nyvad criteria for caries assessment in primary teeth. Eur J Oral Sci 2011; 119: 225-231.

22. Braga MM, Ekstrand KR, Martignon S, Imparato JCP, Ricketts DNJ, Mendes FM. Clinical performance of two visual scoring systems in detecting and assessing activity status of occlusal caries in primary teeth. Caries Res 2010; 44: 300-308.

23. Slayton RL, Urquhart O, Araujo MWB, et al. Evidence-based clinical practice guideline on nonrestorative treatments for carious lesions: a report from the American Dental Association. J Am Dent Assoc 2018; 149: 837-849.e19.

24. Lin YS, Rothen ML, Milgrom P. Pharmacokinetics of 38\% topical silver diamine fluoride in healthy adult volunteers. J Am Dent Assoc 2019; 150: 186-192. 
25. Espinosa-Cristóbal LF, Martínez-Castañón GA, Martínez-Martínez RE, et al. Antibacterial effect of silver nanoparticles against Streptococcus mutans. Mater Lett 2009; 63: 2603-2606.

26. Pérez-Díaz MA, Boegli L, James G, et al. Silver nanoparticles with antimicrobial activities against Streptococcus mutans and their cytotoxic effect. Mater Sci Eng C 2015; 55: 360-366.

27. Noronha VT, Paula AJ, Duran G, et al. Silver nanoparticles in dentistry. Dent Mater 2017; 33: 1110-1126.

28. Tran HV, Dai Tran L, Ba CT, et al. Synthesis, characterization, antibacterial and antiproliferative activities of monodisperse chitosanbased silver nanoparticles. Colloids Surfaces A Physicochem Eng Asp 2010; 360: 32-40.

29. Zhi QH, Lo ECM, Lin HC. Randomized clinical trial on effectiveness of silver diamine fluoride and glass ionomer in arresting dentine caries in preschool children. J Dent 2012; 40: 962-967.

30. Mattos-Silveira J, Floriano I, Ferreira FR, Viganó MEF, Mendes FM, Braga MM. Children's discomfort may vary among different treatments for initial approximal caries lesions: preliminary findings of a randomized controlled clinical trial. Int J Paediatr Dent 2015; 25: $300-304$. 